\title{
Effects of platelet-rich plasma on the activity of human menstrual blood-derived stromal cells in vitro
}

\author{
Siwen Zhang ${ }^{1}$, Pingping $\mathrm{Li}^{1}$, Zhengwei Yuan ${ }^{2}$ and Jichun $\operatorname{Tan}^{1 *}$ (])
}

\begin{abstract}
Background: Human menstrual blood-derived stromal cells (MenSCs) are highly proliferative and show multiple differentiation capacity. The convenience and non-invasiveness make MenSC a novel cell source for regenerative medicine applications. Platelet-rich plasma (PRP) contains abundant growth factors which are beneficial to wound healing. However, the influence of PRP on MenSCs remains elusive. Here, we evaluated the role of PRP in MenSCs proliferation and assessed the effects of PRP on endometrial receptivity regulation in vitro.

Methods: MenSCs cultured with 10\% activated PRP were compared with those cultured with 10\% fetal bovine serum (FBS). Differences in cell proliferation, differentiation, and endometrial receptivity-related gene expression were evaluated.

Results: Notably, 10\% activated PRP significantly promoted MenSCs proliferation and adipogenic/osteogenic differentiation while suppressing apoptosis. Expression of the mesenchymal stem cells (MSCs) marker CD105 and the perivascular markers SUSD2 and CD146 were elevated after PRP treatment. Moreover, short-term PRP stimulation activated the phosphorylation of Akt and signal transducer and activator of transcription 3 (STAT3) pathways, upregulated expression of FoxO1, LIF, and ILT- $\beta$, and downregulated IL-6.

Conclusions: In summary, PRP could promote MenSC proliferation, markedly accelerate cell stemness, and evaluate MenSC functions by enhancing the expression of angiogenesis and endometrial receptivity markers, suggesting its potential use as a promising supplement for MenSCs in endometrial regenerative medicine. Our results provide a theoretical basis for the clinical application of co-transplantation of PRP combined with MenSCs.
\end{abstract}

Keywords: Menstrual blood-derived stromal cells, Platelet-rich plasma, Endometrial receptivity, MenSCs

\section{Background}

Menstrual blood-derived stromal cells (MenSCs) are isolated human endometrial stem cells (hENSCs) obtained during menstrual shedding [1]. MenSCs are located in the basalis and functionalis of the endometrium and are thought to play a key role in endometrial regeneration [2]. The perivascular markers CD146, platelet-derived growth factor receptor (PDGFR)- $\beta$, and sushi domaincontaining 2 (SUSD2) can be used as specific markers for isolation of MenSCs directly from the endometrial shedding mixture by flow cytometry [3]. Moreover, cells

\footnotetext{
*Correspondence: tjczjh@163.com

${ }^{1}$ Reproductive medicine Center, Obstetrics and Gynecology Department, Shengjing Hospital affiliated to China Medical University, No. 39 Huaxiang Road, Tiexi District, Shenyang 110022, China

Full list of author information is available at the end of the article
}

with high expression of SUSD2 or co-expression of SUSD2 and CD146 generate more colony-forming units (CFUs) than the CD146 ${ }^{+}$PDGFR- $\beta^{+}$subpopulation in the proliferative endometrium, suggesting that these stem cells have a role in functionalis growth [4]. In addition to the basic characteristics of mesenchymal stem cells (MSCs), i.e., self-renewal, clonogenicity, and multipotency, MenSCs exhibited higher extraction efficiency and longer passaging capacity [5]. Moreover, MenSCs show improved proliferation capability with a shorter population doubling time of $20 \mathrm{~h} \mathrm{[6],} \mathrm{which} \mathrm{is} \mathrm{twofold} \mathrm{faster}$ than that of bone marrow-derived mesenchymal stromal cells (BM-MSCs).

In previous studies, neither tumor growth nor toxicity was detected after MenSCs treatment in animal models. 
Moreover, the injection of MenSCs resulted in substantial inhibition of tumor development [7]. Recent reports have shown the potential clinical applications of MenSCs in the treatment of type 1 diabetes mellitus [6], stroke [8], ovarian failure [9], Asherman's syndrome [10], and epithelial ovarian cancer [11]. In our previous study, we transferred autologous MenSCs to seven infertile women who were diagnosed with severe Asherman's syndrome and demonstrated that the endometrial thickness of all patients was significantly increased, including in one patient who had an ongoing pregnancy. Our results suggest that MenSCs transplantation may be a promising option for endometrial regeneration and improvement of endometrial receptivity improvement [10].

Current concerns with stem cell-based therapy focus on the surgical procedures involved in sample collection. Compared with other MSCs, MenSCs are free of ethical concerns and can be harvested easily and noninvasively from menstrual blood, therefore, making them attractive for applications in regenerative medicine [7, 12]. Generally, fetal bovine serum (FBS) is the most common medium supplement for therapeutic stem cell culture in vitro. However, the use of FBS-based culture is associated with the risk of infectious disease and immunological reaction during cell transplantation treatment [13].

Human platelet-rich plasma (PRP) is an artificial blood-derived product with the significant advantage of enabling autologous transplantation. In small volumes of plasma, the density of platelets in PRP is 5-10 times to that in whole blood. Platelets are commonly concentrated from the peripheral blood via two or three steps using different centrifugal forces or via mechanical collection [14]. The active ingredient, platelets, are rich in growth factors (GFs) and cytokines, including vascular endothelial growth factor (VEGF), transforming growth factor $\beta-1$ (TGF $\beta-1)$, platelet-derived growth factor (PDGF), epidermal growth factor (EGF), hepatocyte growth factor (HGF), and insulin-like growth factor (IGF-1) [15]. These therapeutic molecules are released after platelet activation and are beneficial for cell proliferation and wound healing [16]. Moreover, by serving as a cellular scaffold, PRP boosts the potency of transplanted cells used in stem cell-based therapies [17]. In vitro, PRP has been clearly demonstrated to increase cell biological functions. Adult cells, such as epithelial cells, chondrocytes and osteoblasts as well as multipotent cells, such as adipose tissue-derived stem cells (ADSCs), BM-MSCs, and tendon stem cells have been reported to show enhanced proliferation after PRP treatment [18-20]. Besides promotion of proliferation, differentiation, and recruitment are also observed, suggesting potential beneficial effects in tissue regeneration [21]. Moreover, human platelet products have been shown to be more effective in induction of osteogenic lineage differentiation of MenSCs compared with FBS-containing culture medium [22, 23]. In addition to basic nutritional support, PRP has also been shown to be a promising substitution for FBS in cell culture because of its self-collectivity, low cost and low immunogenicity [23].

In this study, we aimed to evaluate the effects of PRP on the regulatory effects of MenSCs on biological and endometrial function in vitro. Our results provide important insights into the mechanisms through which PRP affects the activity of MenSCs in vitro and the value of PRP in autologous transplantation for improvement of endometrial receptivity.

\section{Methods}

MenSCs isolation and culture

Six menstrual blood donors were diagnosed without reproductive system diseases, aged 25-35 years old. All donors gave consent and all procedures were approved by the Ethics Committee of Shengjing Hospital affiliated to China Medical University (2017PS330K).

MenSCs were collected and individually cultured according to our previous report [10]. Briefly, after vagina disinfection, $5 \mathrm{ml}$ samples of menstrual blood were collected on day 2 of menses with a $20-\mathrm{ml}$ injection syringe. Then the menstrual blood was transferred onto Ficoll (Sigma-Aldrich, St. Louis, MO, USA), fractionated at a density-gradient centrifugation according to the manufacturer's instructions. The central cell layer with purified mononuclear cells were washed and cultured in $25-\mathrm{cm}^{2}$ tissue culture bottles (NEST) with Chang's medium: Dulbecco's modified Eagle medium: nutrient mixture F-12 (Ham's) (1:1, HyClone, Logan, UT, USA) with 10\% FBS (Gibco, Waltham, MA, USA) and 1\% penicillin-streptomycin (Sigma-Aldrich). In a humidified incubator, cells were grown under $37{ }^{\circ} \mathrm{C}$ in an atmosphere containing $5 \% \mathrm{CO}_{2}$. After $24 \mathrm{~h}$ the medium was changed and the attached cells were washed by phosphate-buffered saline (PBS). The culture medium was changed every 3 days until adherent cells reach 80 $90 \%$ confluence, then the cells were passaged by $0.25 \%$ trypsin (Sigma-Aldrich).

\section{Platelet-rich plasma (PRP) preparation}

Platelet-rich plasma was extracted from apheresis platelets (a platelet-plasma mixture with a concentration of $12.5 \times 10^{11} / \mathrm{L}$, from a healthy female donor aged 30 ), acquired from the blood transfusion department of Shenging Hospital. 20\% $\mathrm{CaCl}_{2}$ (Sigma-Aldrich) plus 1000 $\mathrm{U} / \mathrm{ml}$ thrombin from bovine plasma (T8020, Solarbio, Beijing, China) was used for activation, added into the apheresis platelets at a volume ratio of 1:20. The mixture was incubated at $37{ }^{\circ} \mathrm{C}$ for $1 \mathrm{~h}$ and at $4{ }^{\circ} \mathrm{C}$ for $12 \mathrm{~h}$. For collection of activated PRP, the gel-like mixture was centrifuged at $5000 \mathrm{rpm}$ for $30 \mathrm{~min}$ at $4{ }^{\circ} \mathrm{C}$. The supernatant 
was aspirated and filtered through $0.22-\mu \mathrm{m}$ filters and then aliquoted and stored at $-80{ }^{\circ} \mathrm{C}$, to avoid repeated freezing and thawing.

\section{Cell proliferation assay}

Cell-counting kit 8 (CCK-8) cell proliferation assay was performed by cell counting kit-8 (CCK8; Dojindo, Kumamoto, Japan) for 7 days. We set the culture medium with gradient concentrations of activated PRP $(20 \%, 10 \%$, $5 \%, 2.5 \%$ ) as experimental groups, $10 \% \mathrm{FBS}$ as the control group and serum-free medium as the blank control group. P4 MenSCs were seeded onto 96-well plates at approximately $2 \times 10^{3}$ cells per well with Chang's medium. After $24 \mathrm{~h}$, the original medium was exchanged as grouped and refreshed every 3 days. Every day at an indicated time, wells containing cells were washed once by PBS then 100 $\mu \mathrm{l}$ DMEM and $10 \mu \mathrm{l}$ CCK8 was added to cells, incubated at $37{ }^{\circ} \mathrm{C}$ for $2.5 \mathrm{~h}$. Optical density (OD) was measured by $450 \mathrm{~nm}$ absorbance. A MTS (Promega, Beijing, China) assay (for 7 days) was performed as a verification of CCK8 assay. $20 \mu \mathrm{l}$ MTS was added to cells and incubated for $2.5 \mathrm{~h}$. The results are shown in Additional file 1: Data 1.

\section{EdU proliferation assay}

EdU assay was performed to certificate the proliferation promotion effect of PRP. We assessed 10\% PRP as the experiment group and 10\% FBS as the control. P4 $4 \times 10^{3}$ MenSCs were plated onto 96-well plates per well. The medium was changed into serum-free medium overnight when the cells grew to a density of $40 \%$. After that the cells were incubated by PRP or FBS for 24 and $48 \mathrm{~h}$, then they were disposed as per the EdU manufacturer's instructions (C00031, Riobio, Guangzhou, China). The immunoflurorescence was captured by Nikon Eclipse Ni (Nikon, Tokyo, Japan), the positive rate was analyzed by Image Pro Plus 6 (Media Cybernetics Rockville, MD, USA).

\section{Flow cytometry}

P4 MenSCs were maintained in 100-mm culture dishes (BD Falcon, BD Biosciences, San Jose, CA, USA) for flow cytometry. Briefly, after being incubated in serum-free medium overnight to synchronize the cell cycle, cells were cultured with $10 \%$ PRP or $10 \%$ FBS for $24 \mathrm{~h}$ or $48 \mathrm{~h}$. Then the cells were collected and tested.

We measured surface makers CD34, CD38, CD44, CD45, CD73, CD90, CD105, SSEA-4, CD146, and SUSD2 after 24-h incubation. $1 \times 10^{6}$ cells were suspended for $100 \mu \mathrm{l}$ in $1 \%$ BSA/PBS incubated with antibodies of CD34-FITC, CD38-PE, CD44-FITC, CD45-FITC, CD73PE, CD90-PE, CD105-FITC, or SSEA-4-PE (BD, Franklin Lakes, NJ, USA). CD146-FITC and SUSD2-PE (Biolegend, San Diego, CA, USA) were co-incubated. For apoptosis analysis, $1 \times 10^{6}$ cells were stained with Annexin V-FITC/ PI kit following the manufacturer's protocol (\#88-8007,
eBioscience, San Diego, CA, USA). Briefly, the cells were washed twice by PBS and suspended with $100 \mu$ l binding buffer, $5 \mu \mathrm{l}$ Annexin V-FITC was added into each tube. After incubated in the dark for $15 \mathrm{~min}$, the cells were washed then diluted to $200 \mu \mathrm{l}$ with $5 \mu \mathrm{l}$ propidium iodide (PI), and analyzed by flow cytometry. For cell cycle analysis, cells of each group were fixed with $70 \%$ ethanol-PBS at $4{ }^{\circ} \mathrm{C}$ for $24 \mathrm{~h}$, then be washed and suspended with 300 $\mu \mathrm{l}$ PBS. The suspension was treated with $3 \mu \mathrm{l}$ RNase A (10 $\mathrm{mg} / \mathrm{ml}$, Solarbio) and $3 \mu \mathrm{l} \mathrm{PI}, 10 \mathrm{mg} / \mathrm{ml}$ (Solarbio) and washed in water at $37{ }^{\circ} \mathrm{C}$ for $30 \mathrm{~min}$. These cells were analyzed by fluorescence-activated cell sorting using a flow cytometer (BD FACSCalibur; BD Biosciences). The rates of apoptosis and phenotype were analyzed by CellQuest, cell cycle was quantified by ModFit LT for Mac v3.0 (BD Biosciences).

\section{Mesenchymal stem cell differentiation properties}

To assess the influence of PRP on MenSCs differentiation, we cultured P4 MenSCs in adipogenic and osteogenic induction medium with $10 \%$ activated PRP or $10 \%$ FBS.

We seeded over $10^{5}$ MenSCs in $35-\mathrm{mm}$ culture plates pre-incubated with Chang's medium, setting PRP treatment as the experimental groups and FBS treatment as the control groups. When cell density reached $30 \%$, the osteogenic induction began (ascorbic acid $2 \mu \mathrm{l} / \mathrm{ml}$, betaglycerophosphate sodium $10 \mu \mathrm{l} / \mathrm{ml}$, dexamethasone 0.1 $\mu \mathrm{l} / \mathrm{ml}$ ) while the density reached $100 \%$ for adipogenic treatment (medium A: insulin $2 \mu \mathrm{l} / \mathrm{ml}$, IBMX $1 \mu \mathrm{l} / \mathrm{ml}$, rosiglitazone $1 \mu \mathrm{l} / \mathrm{ml}$, dexamethasone $0.1 \mu \mathrm{l} / \mathrm{ml}$; medium B: insulin $2 \mu \mathrm{l} / \mathrm{ml})$. All the cells were cultured in induction medium (Cyagen, Guangzhou, China) for 3 weeks (six cycles) at $37{ }^{\circ} \mathrm{C}$ in $5 \% \mathrm{O}_{2} / 5 \% \mathrm{CO}_{2} / 90 \% \mathrm{~N}_{2}$, medium changed as protocol. Then the cells were fixed and specifically colored to detect the differentiation: Oil Red for adipogenesis and Alizarin Red for osteogenesis (Cyagen). Stained cells were examined and photographed using Nikon ECLIPSE Ni (Nikon).

\section{Immunofluorescence microscopy}

P4 MenSCs were cultured on coverslips (14 mm, NEST) at $5 \times 10^{4}$ cells $/ \mathrm{ml}$ in 12 -well plates. After overnight starvation, the medium was exchanged to $10 \%$ PRP or $10 \%$ FBS-DMEM/F12 incubated for $24 \mathrm{~h}$. Then the coverslips were washed twice by PBS and fixed in $4 \%$ PFA, followed by protein block (SP-9001, ZSGB-BIO) for 30 min at $37^{\circ} \mathrm{C}$. Purified SUSD2 antibody (1:100, \#327401, BioLegend), CD146 antibody (1:100, ab75769, Abcam, Cambridge, MA, USA), Vimentin antibody $(1: 100$, D21H3, CST), cytokeratin 18 (CK18) antibody (1:100, ab181597, Abcam) diluted in PBS were immunostained overnight at $4{ }^{\circ} \mathrm{C}$. Next day, all the coverslips were incubated with secondary antibodies (1:500, Cy3-labled goat anti-mouse IgG, FITC-labeled goat anti-rabbit IgG, Cy3- 
labeled goat anti-rabbit IgG, Beyotime, Beijing, China) at room temperature for $2 \mathrm{~h}$. DAPI (1:20, Beyotime) was used to visualize nuclei. Images were observed and captured using Nikon ECLIPSE Ni (Nikon), the fluorescence was analyzed by DOI/Area using Image Pro-Plus 6 (Media Cybernetics).

\section{Quantitative RT-PCR}

P4 MenSCs were used to extract total RNA with RNAiso Plus (\#9108, Takara, Tokyo, Japan), the expression of FoxO1, HOXA10, LIF, CK18, IL1- $\beta$, IL6, RUNX2, and PPAR 2 was analyzed. Grouped as introduced previously, cells were conditionally cultured for $6 \mathrm{~h}$ or $24 \mathrm{~h}$. Cells that were serum-starved overnight were set as control. The RNA was reverse transcribed into cDNA using PrimeScript RT Regent Kit (\#RR047A, Takara) according to the manufacturer's protocol following by the RT reaction that as $37{ }^{\circ} \mathrm{C}$ for $15 \mathrm{~min}, 85^{\circ} \mathrm{C}$ for $5 \mathrm{~s}$ and $4{ }^{\circ} \mathrm{C}$. All the cDNA was stored at $-20{ }^{\circ} \mathrm{C}$. Quantitative PCR was performed using SYBR Premix Ex Taq ii (\#RR820A, Takara). The primers used in this study are listed in Table 1. Quantitative RT-PCR was conducted at $95{ }^{\circ} \mathrm{C}$ for $30 \mathrm{~s}$ followed by 40 cycles at $95{ }^{\circ} \mathrm{C}$ for $5 \mathrm{~s}$ and $60{ }^{\circ} \mathrm{C}$ for $34 \mathrm{~s}$ and final extension at $60^{\circ} \mathrm{C}$ for 15 $\mathrm{s}$ in 7500 software v2.0.6 (Life Technologies, Carlsbad, CA, USA). The relative levels of mRNA were normalized with GAPDH, gene expression was analyzed by $2^{-\Delta \Delta \mathrm{Ct}}$.

\section{Western blot}

The treatment and groups of cells were the same as RTPCR treatment. Cells were scraped off the bottles with

Table 1 Quantitative polymerase chain reaction primer sequences

\begin{tabular}{|c|c|}
\hline Name & Sequence $\left(5^{\prime}-3^{\prime}\right)$ \\
\hline \multirow[t]{2}{*}{ FoxO1 } & F: TACGAGTGGATGGTCAAGAGC \\
\hline & R: TGAACTTGCTGTGTAGGGACA \\
\hline \multirow[t]{2}{*}{ HOXA10 } & F: CTGAGGTCAATGGTGCAAAGGA \\
\hline & R: TTGCCAACCTGCATGTCCA \\
\hline \multirow[t]{2}{*}{ LIF } & F: CCAACAGCAAGACGAGGATG \\
\hline & R: GATGAAGCAGGAAGGAGAAGG \\
\hline \multirow[t]{2}{*}{ CK18 } & F: ATCTTGGTGATGCCTTGGAC \\
\hline & R: CTCAGAACTTTGGTGTCATTGG \\
\hline \multirow[t]{2}{*}{$1 L-1 \beta$} & F: TGGCAATGAGGATGACTTGT \\
\hline & R: TGGTGGTCGGAGATTCGTA \\
\hline \multirow[t]{2}{*}{ IL-6 } & F: TTCGGTCCAGTTGCCTTCT \\
\hline & R: GGTGAGTGGCTGTCTGTGTG \\
\hline \multirow[t]{2}{*}{ RUNX2 } & F: GCAGCAGCAGCAGCAGGAG \\
\hline & R: GCACCGAGCACAGGAAGTTGG \\
\hline \multirow[t]{2}{*}{ PPARY2 } & F: AGAACAGATCCAGTGGTTGCAGATTAC \\
\hline & R: CAGACACGACATTCAATTGCCATGAG \\
\hline \multirow[t]{2}{*}{ GAPDH } & F: CAGGAGGCATTGCTGATGAT \\
\hline & R: GAAGGCTGGGGCTCATTT \\
\hline
\end{tabular}

$250 \mu \mathrm{l}$ radio immunoprecipitation assay (RIPA) buffer combined protease inhibitor (PMSF) (P0013B, ST506, Beyotime). The lysates were collected by centrifugation at $14,000 \mathrm{rpm}$ for $20 \mathrm{~min}$ at $4{ }^{\circ} \mathrm{C}$. Total protein was qualified by BCA kit (P0010S, Beyotime), samples were diluted to $2 \mu \mathrm{g} / \mu \mathrm{l}$. Protein samples were separated by $8 \%$ sodium dodecyl sulfate-polyacrylamide gel electrophoresis (Beyotime) and $0.45 \mu \mathrm{m}$ PVDF membranes (EMD Millipore, Billerica, MA, USA) were used for transferred, $5 \%$ non-fat powder milk dissolved in TBST was used for blocking. Thereafter, all membranes were incubated with primer antibodies listed below for $12 \mathrm{~h}$ at $4{ }^{\circ} \mathrm{C}$ (Akt \#4691, 1:1000, Stat3 \#4904, 1:1000, Phosopho-Akt (Thr308) \#13,038, 1:1000, Phosopho-Akt (Ser473) \#4060, 1:1000, Phosopho-Stat3 (Tyr705) \#9145, 1:1000, FoxO1 \#2880, 1:1000, all from Cell Signaling Technology, Danvers, MA, USA; LIF antibody \#abs 120615 from Absin, 1:300; IL1- $\beta$ antibody \#16806-1-AP, 1:500, IL6 antibody \#21865-1-AP,1:500 from (Proteintech, Chicago, IL, USA). Subsequently, the membranes were incubated on the following day with secondary antibody (peroxidaseconjugated affinipure goat anti-rabbit IgG, ZSGB-BIO, Beijing, China) for $1 \mathrm{~h}$ at room temperature. Thereafter, the blots were added ECL (\#1862420, \#1862421, Thermo Fisher Scientific, Waltham, MA, USA) and visualized by C300. The gray densitometric was analyzed using Image J (National Institutes of Health, Bethesda, MD, USA).

\section{Statistics}

Descriptive statistics and statistical analysis were carried out using GraphPad Prism 5 (San Diego, CA, USA). Results are presented as mean \pm SEM. One-way ANOVA test was used in CCK8 and MTS statistical analysis and two-tailed unpaired $t$ test was used in other experiments. ${ }^{*} P<0.05,{ }^{* * *} P<0.01$, ${ }^{* * *} P<0.001$ were considered as statistical significant.

\section{Results \\ PRP promoted MenSCs proliferation while protecting cells from apoptosis}

First, we examined the effects of PRP on MenSCs proliferation using cell counting kit-8 (CCK8) assays $(n=3)$. As shown in Fig. 1a, compared with the serum-free group, activated PRP significantly increased the number of viable cells and showed positive concentrationdependent effects. During the first 3 days, optical density (OD) values in all serum-in groups were nearly identical. Compared with 10\% FBS, groups with PRP showed increased cell proliferation beginning on day 4 , and this effect persisted for days. OD values of $10 \%$ and $20 \%$ activated PRP were significantly higher than those of the $10 \%$ FBS group. Accordingly, all further experiments were conducted with $10 \%$ PRP. The OD values of several groups decreased slightly on day 7 , probably because the 


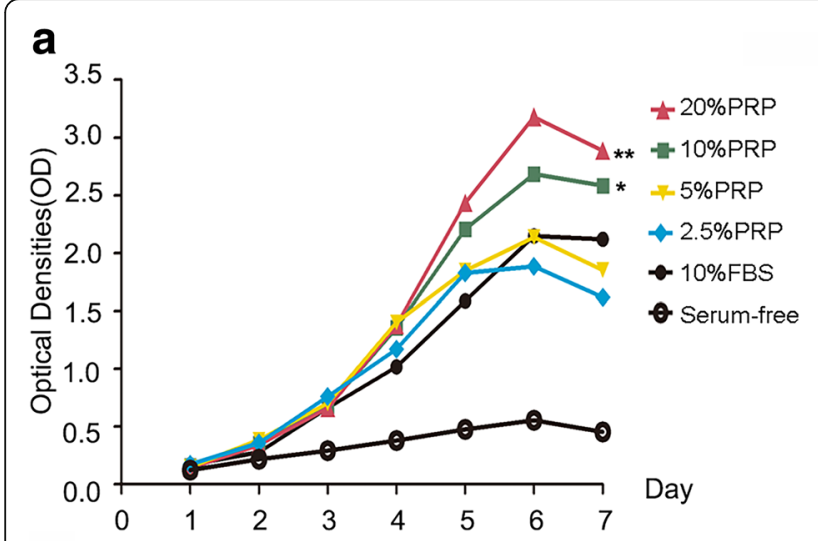

b EdU DAPI Merged
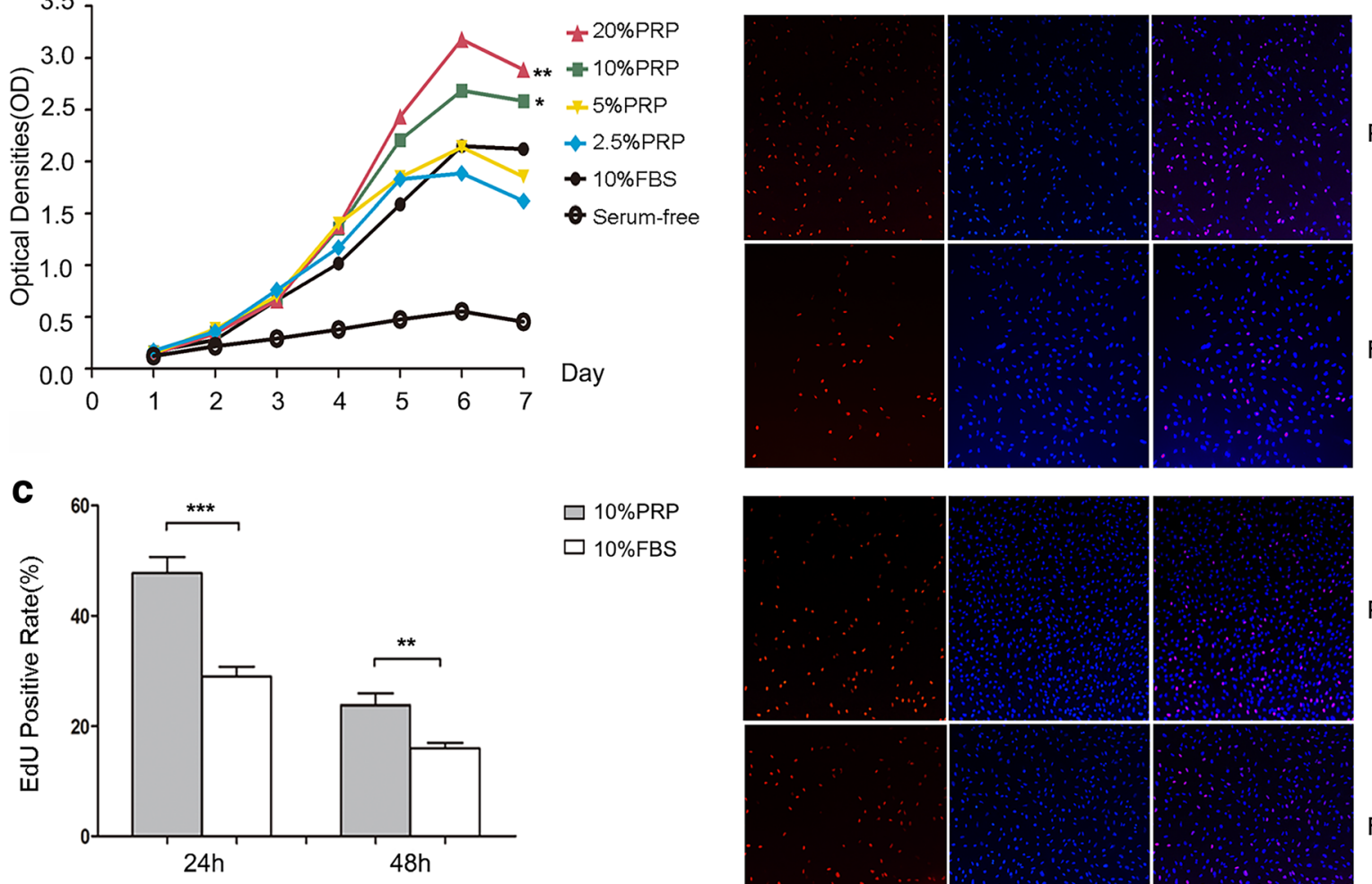

PRP 24h

FBS 24h
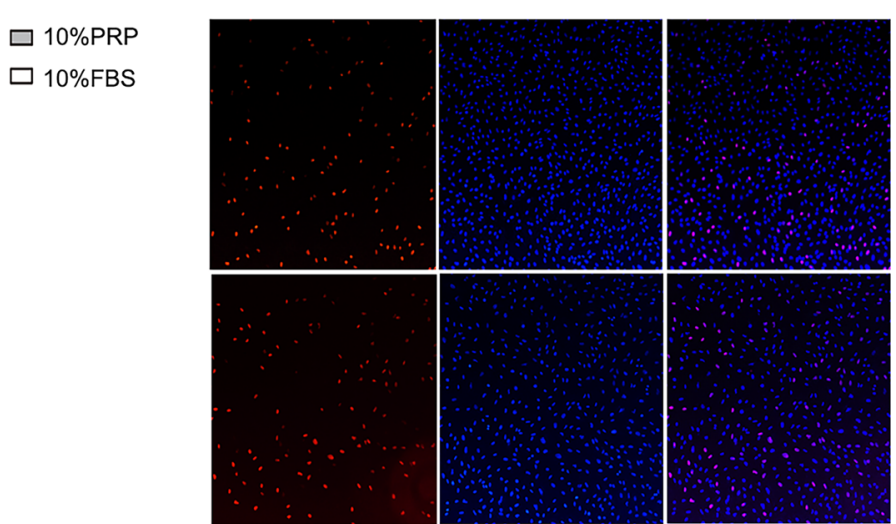

PRP 48h

Fig. 1 PRP promoted MenSCs proliferation. a CCK8 assay detected proliferation of P4 MenSCs cultured with different concentrations of activated PRP or 10\% FBS $(n=3)$. $\mathbf{b}$ Immunofluorescence analysis of EdU+ MenSCs under stimulation of 10\% PRP and 10\% FBS for $24 \mathrm{~h}$ and $48 \mathrm{~h}$ (red: DNA replicating, blue: nuclei, $n=6$ ). c Statistical analysis of EdU+ cell rate of each group. MenSCs cultured with 10\% PRP showed higher proliferation rate for both $24 \mathrm{~h}$ and $48 \mathrm{~h}(P<0.01)$. CCK8 was analyzed by one-way ANOVA test. Data was mean $\pm \mathrm{SEM},{ }^{*} P<0.05,{ }^{* *} \mathrm{P}<0.01,{ }^{* * *} P<0.001$ for two-tailed $t$ test

cells were confluent. MTS assays showed the same trend as CCK8 results (Additional file 1: Data 1).

Furthermore, we assessed cell proliferation using 5ethynyl-2'-hydeoxyuridine (EdU, $n=6$ ), a thymidine analog that can permeate into DNA molecules at the $S$ stage instead of thymidine when diluted in culture medium. As shown in Fig. 1b and c, more EdU-positive cells were found in the PRP groups than in the FBS groups $(P=0.0003$ and 0.0022 for 24 and $48 \mathrm{~h}$, respectively). Generally, the positive rate was much higher after $24 \mathrm{~h}$ of culture than after $48 \mathrm{~h}$ of culture in both groups. These results indicated that PRP acted positively on DNA replication in MenSCs, suggesting that PRP may elicit stronger effects after a shorter incubation.

Subsequently, to explore the mechanisms involved in PRP-dependent MenSC proliferation, we detected the cell cycle of both groups using flow cytometry $(n=6)$. As shown in Fig. 2a, after treatment with PRP for $24 \mathrm{~h}$, there was a significant increase in the number of cells in the $\mathrm{S}$ phase $(P=0.029)$ compared with those in the FBS group.
There were no differences between the two groups for cells in the $G_{0} / G_{1}$ phase or $G_{2} / M$ phase. Moreover, as shown in Fig. 2b, early (Annexin $\mathrm{V}^{+} \mathrm{PI}^{-}$), late (Annexin $\mathrm{V}^{+} \mathrm{PI}^{+}$) and total apoptosis (Annexin $\mathrm{V}^{+}$) in $\mathrm{P} 4$ MenSCs were significantly inhibited after PRP treatment for $24 \mathrm{~h}(P=0.0376, P$ $=0.0178$ and $P=0.0079$, respectively, $n=6$ ) as compared with the control. However, at $48 \mathrm{~h}$, the differences were not significant. These results indicated that PRP promoted MenSC proliferation by upregulation of DNA replication and downregulation of cell apoptosis.

\section{PRP elevated the pluripotency of MenSCs}

Flow cytometry was applied to evaluate changes in stemness markers of MenSCs following treatment with PRP $(n=6)$. As shown in Fig. 3a, there were no significant differences in the expression of CD34, CD38, CD44, CD45, CD73, CD90, and SSEA-4 after PRP treatment for $24 \mathrm{~h}$. However, the expression of CD105, which participated in angiogenesis, was upregulated following PRP treatment $(P=0.0381)$. PRP treatment also increased the 


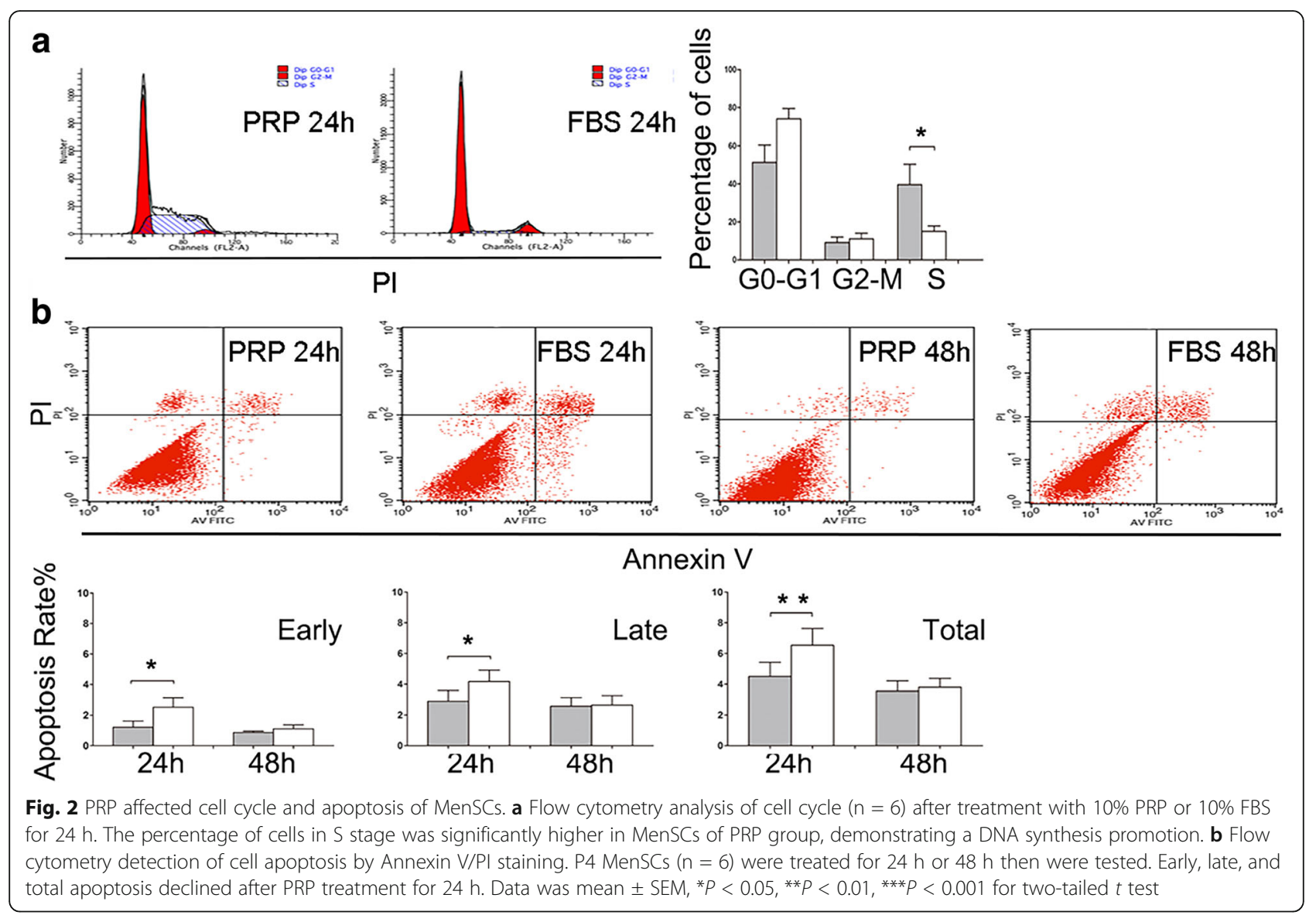

co-expression of perivascular selection markers CD146 and SUSD2 ( $P=0.0493, n=4$; Fig. $3 c)$.

Consistent with these findings, immunofluorescence also showed increased SUSD2 and CD146 expression in MenSCs in the PRP group $(n=6)$. As shown in Fig. 3b, the intensities of SUSD2 and CD146 were significantly increased in the PRP-treated group $(P=0.0489$ and 0.0238 , respectively). The expression of vimentin, a stromal cell marker, and cytokeratin 18 (CK18), an epithelial cell marker, were further analyzed $(n=6)$. Reduced expression of vimentin $(P=0.0117)$ was found in the PRP group, suggesting the role of PRP in maintaining the pluripotency of MenSCs. In contrast, the intensity of CK18 was weak in both groups and showed no significant difference.

To determine whether PRP affected the differentiation capacity of MenSCs, we treated P4 MenSCs with osteogenic or adipogenic induction medium for about 21 days (six cycles according to the protocol; Cyagen) with $10 \%$ PRP or $10 \%$ FBS $(n=3)$. After treatment with PRP plus osteogenic induction medium, MenSCs exhibited strong Alizarin Redstained calcium nodules, with greater amounts than the control (Fig. 3d, left). For adipogenic differentiation, cells treated with PRP plus induction medium showed greater Oil Red O staining (Fig. 3d, right), indicating more lipid droplet formation than cells in the FBS group.
PRP regulated the expression of endometrial receptivityand inflammation-related genes and proteins in MenSCs To ensure the efficiency of PRP-assisted MenSCs in the treatment of endometrial damage, real-time polymerase chain reaction was used to evaluate the expression of endometrial receptivity- and inflammation-related genes in MenSCs cultured with PRP for 6 or $24 \mathrm{~h}(n=6)$. As shown in Fig. 4, FoxO1 expression was higher in PRPtreated cells than in FBS-treated cells at $6 \mathrm{~h}(P=$ 0.0105). Similarly, LIF gene expression was significantly increased in PRP-treated cells compared with that in FBS-treated cells at $6 \mathrm{~h}(P=0.044)$, while weak expression was detected in both groups at $24 \mathrm{~h}$. In contrast, the expression of $H O X A 10$ was significantly decreased following PRP treatment at both 6 and $24 \mathrm{~h}$, compared with that after FBS treatment $(P=0.02$ for $6 \mathrm{~h})$. After PRP treatment, IL1- $\beta$ expression was upregulated at $6 \mathrm{~h}$ and $24 \mathrm{~h}(P=0.0429$ and 0.0497$)$ and IL6 was downregulated $(P=0.0336)$ at $6 \mathrm{~h}$. CK18 gene expression was upregulated, albeit not significantly, at both $6 \mathrm{~h}$ and 24 h. No significant differences in RUNX2 or PPAR 2 (osteogenic and adipogenic genes) were detected at both $6 \mathrm{~h}$ and $24 \mathrm{~h}$.

Western-blot for FoxO1 showed the same trends as observed for mRNA expression $(P=0.0422$ at $6 \mathrm{~h})$ and 


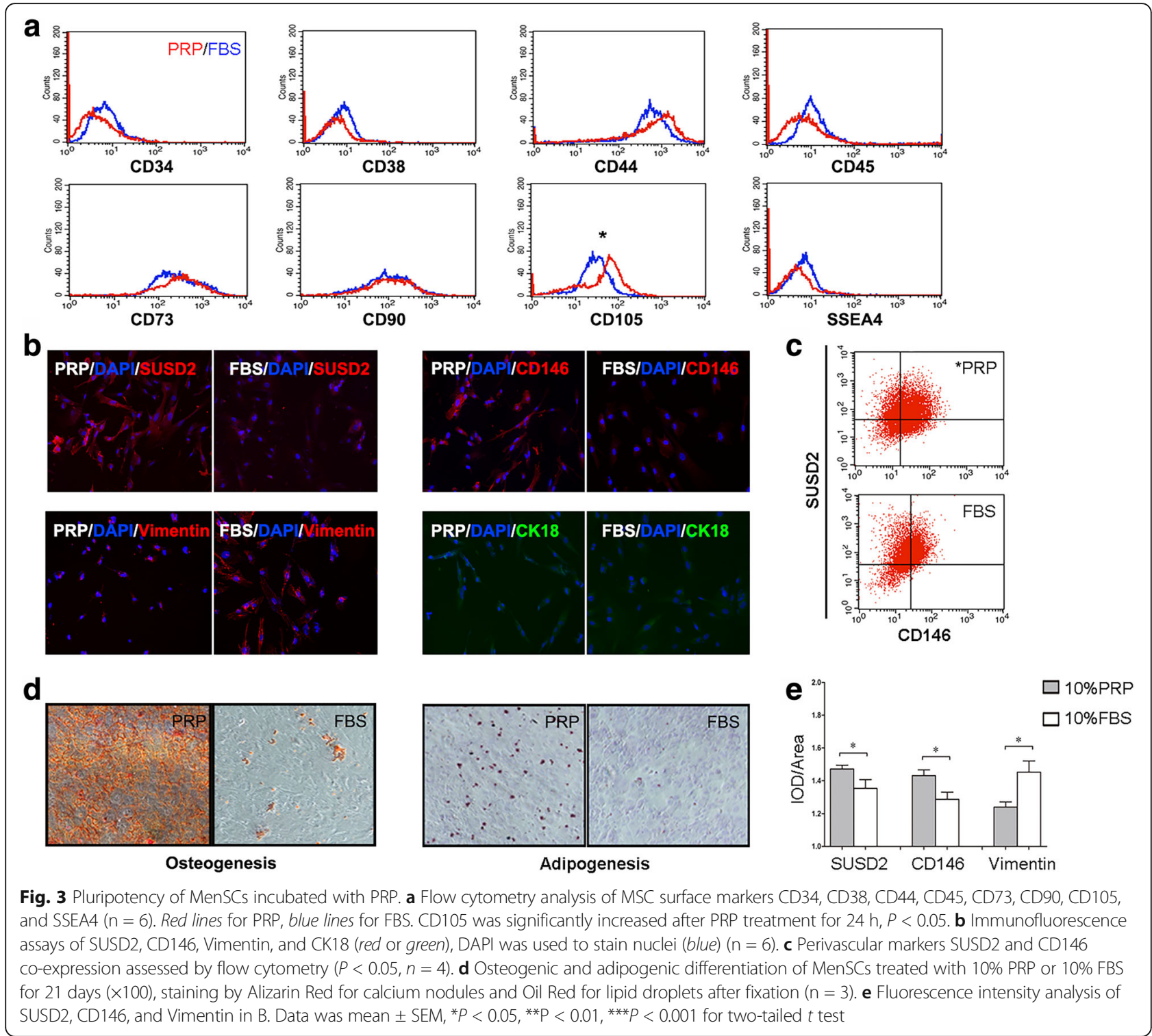

LIF was increased significantly at $6 \mathrm{~h}$ for PRP incubation $(P=0.0096)$. After PRP treatment, IL1- $\beta$ levels were increased at $6 \mathrm{~h}$ and $24 \mathrm{~h}(P=0.0141$ and 0.031$)$ and IL6 level was decreased at $6 \mathrm{~h}(P=0.0286)$ (Fig. $5 \mathrm{a}$ and b).

These data demonstrated that PRP could stimulate the expression of endometrial receptivity- and inflammationrelated genes and proteins.

PRP activated Akt and signal transducer and activator of transcription 3 (STAT3) pathways in MenSCs

We then analyzed the expression levels of proteins in the Akt and STAT3 pathways in MenSCs after PRP treatment for 6 or $24 \mathrm{~h}(n=6)$. As shown in Fig. $5 \mathrm{a}$ and b, phosphoAkt (Thr308) and phospho-STAT3 (Tyr305) levels were significantly increased after PRP induction $(P=0.0002$ and 0.001 for phospho-Akt (Thr308) at 6 and 24 h, respectively; $P=0.0003$ and 0.0032 for phospho-STAT3 (Tyr305) at 6 and $24 \mathrm{~h}$, respectively). Levels of phosphoAkt (Ser473) were also enhanced in the PRP group $(P=$ 0.0084 and 0.0079 at 6 and $24 \mathrm{~h}$, respectively). The expression of total Akt was reduced in the PRP group, which indicated that the increased phospho-Akt could be at least partly explained by increased phosphorylation of AKT.

\section{Discussion}

MenSCs are a type of MSCs that exhibit all generalized MSC characteristics and have several advantages over MSCs. In contrast to BM-MSCs or ADSCs, the process of harvesting MenSCs is completely non-invasive. In addition, MenSCs have a shorter population doubling time and do not show changes in traits according to donor age during early passages (up to passage 10) [24]. 

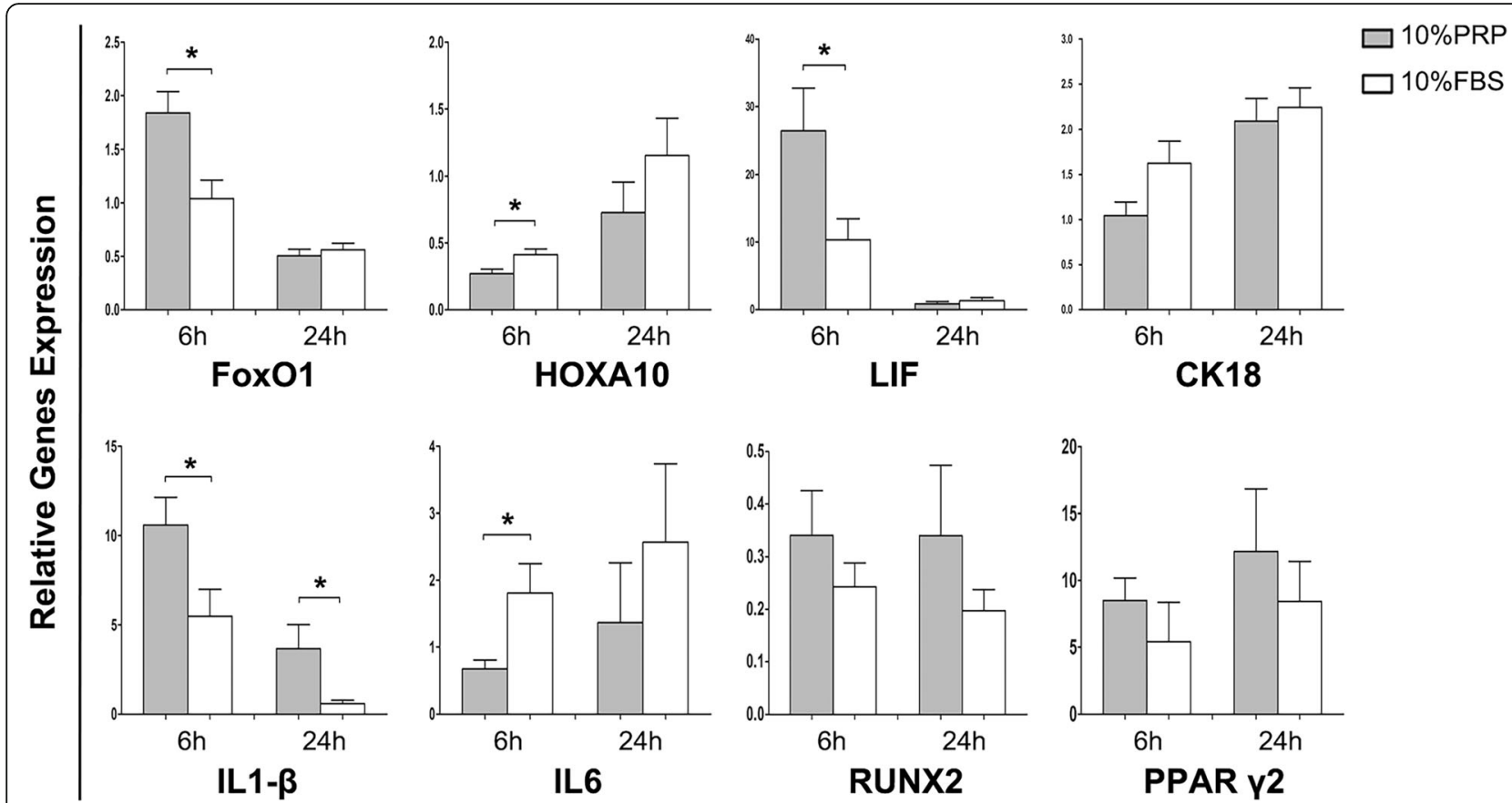

Fig. 4 Quantitative RT-PCR analysis of receptivity- and inflammation-related genes. Gene expression analyzed by RT-PCR. P4 MenSCs ( $n=6$ ) cultured with 10\% PRP (gray) or 10\% FBS (white) for $6 \mathrm{~h}$ and $24 \mathrm{~h}$. RT-PCR analysis of FoXO1, HOXA10, LIF, CK18, IL1- $\beta$, IL6, RUNX2, and PPARY2. GAPDH was used for mRNA standard, fold changes were measured by $2^{-\Delta \Delta C T}$. Data was mean \pm SEM, ${ }^{*} P<0.05$, ** $P<0.01$, ${ }^{* * *} P<0.001$ for two-tailed $t$ test

As a novel type of graft in regenerative medicine, MenSCs have been safely used in the treatment of several diseases based on its low immunogenicity [25].

In this study, we evaluated the effects of human PRP, a promising autologous product, on MenSCs. Several studies [26] have clearly demonstrated that activated PRP promotes the proliferation of many MSCs lines in a concentration-dependent manner in vitro. Consistent with these findings, our study revealed that higher concentrations of activated PRP had stronger effects for MenSCs culture duration. According to the results of CCK8 assays, we concluded that $10 \%$ activated PRP was the optimal concentration for MenSCs proliferation, similar to the results of a previous study [27], in which 10\% PRP was found to have the greatest effects on the proliferation of tendon progenitor cells. In this study, activated PRP increased the ratio of cells in the $S$ phase and inhibited apoptosis, probably via activation of the Akt and IL-6/ STAT3 pathways. These results showed that the growth factors released by PRP accelerated DNA replication in MenSCs and promoted cell proliferation.

PRP is a potential growth factor supplement that has been suggested to act as a catalyst and to accelerate MSC differentiation without affecting cellular structure and biology affects. Abundant GFs in PRP such as PDGF, IGF, and TGF- $\beta$ have been reported to have stimulatory effects on stem cell differentiation [28]. According to a previous study on BM-MSC, RUNX2, and PPAR $\gamma 2$, the master genes expression for osteogenic and adipogenic genes were slightly upregulated after treatment with PRP releasate for 5 days [21]. Similar results were also detected in ADSC osteogenic differentiation after PRP treatment for 21 days [23]. General mineralization markers such as Alizarin Red and alkaline phosphatase were also increased as a result of PRP addition, suggesting that osteoblast differentiation and matrix mineralization may be modulated by RUNX2 expression [29]. Similar results were also detected in ADSCs after PRP treatment for 21 days [19]. However, according to a study by Amable, no significant differences in adipogenic gene expression were detected in differentiated BM-MSCs with PRP incubation for 21 days, although the stimulatory effects of PRP on adipogenic differentiation were obvious [30]. The results of the present study showed that $10 \%$ activated PRP promoted osteogenesis and adipogenesis in MenSCs (21 days). However, no significant differences in related gene expression were detected at $6 \mathrm{~h}$ or $24 \mathrm{~h}$, probably because of the short incubation time. Further studies are needed to elucidate the mechanisms through which PRP affects MenSC differentiation.

In this study, the PRP we used was extracted from apheresis platelets, similar to clinical autologous pure PRP (P-PRP) separated from leukocytes and proinflammatory cytokines. P-PRP has been shown to be more suitable for bone regeneration and acute tendon injury healing [31], indicating an anti-inflammatory effect. A previous study demonstrated that PRP provides 


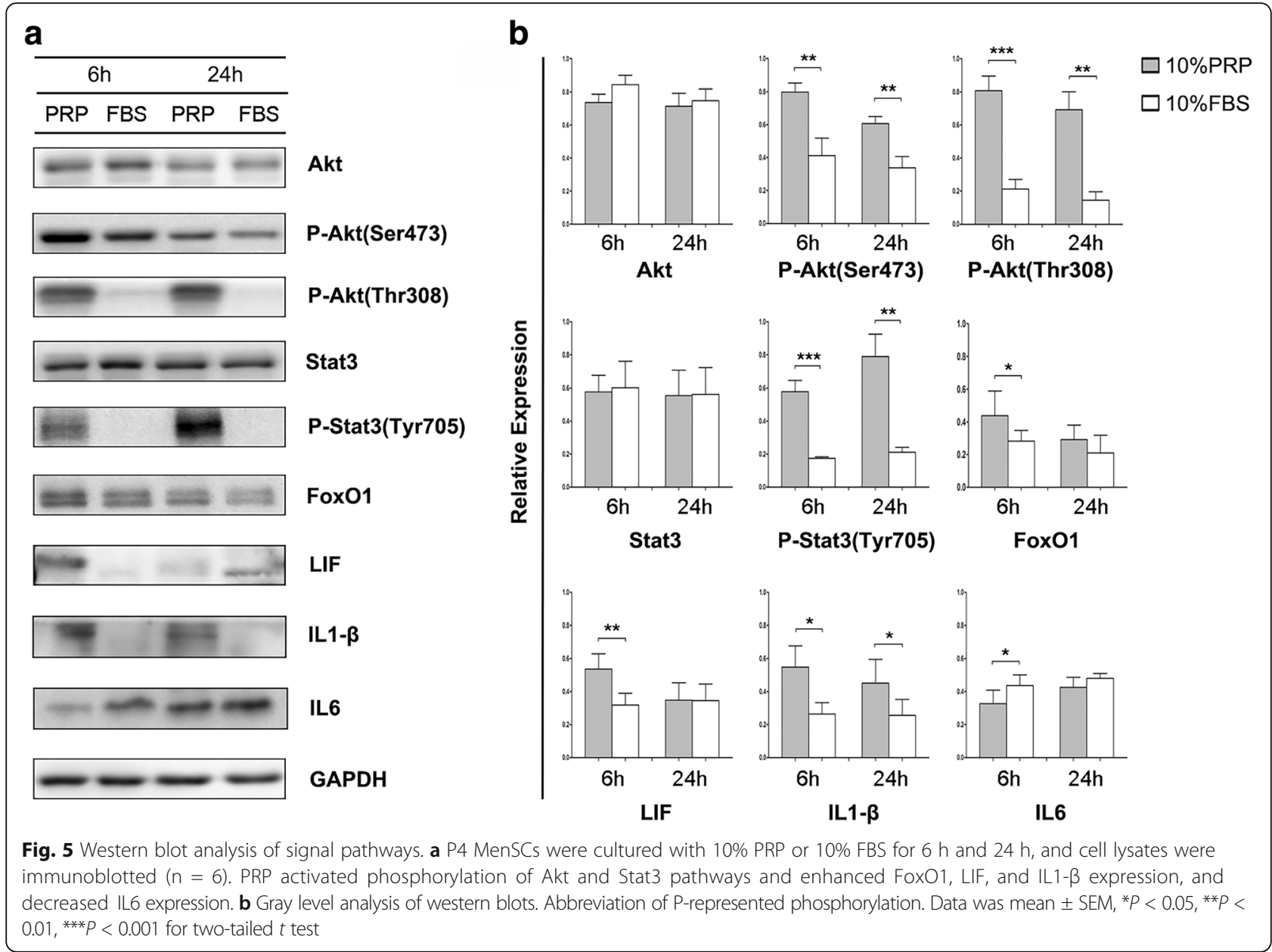

the immunoregulatory effects of downregulating crucial inflammatory cytokines, such as interleukin IL-6 and IL-8. IL6 is a pro-inflammatory cytokine and participated in the implantation window. It is essential to form an antiinflammatory state following implantation in order to prevent fetal rejection [32]. In this study, it was demonstrated that the expression of IL-6 was downregulated in MenSCs treated with PRP compared with that in cells treated with FBS, suggesting an anti-inflammatory effect in endometrium. Another pro-inflammatory cytokine, IL1- $\beta$, was upregulated after PRP incubation. IL1- $\beta$ is also known to be increased in the mid-secretory phase of human endometrium, which is essential for embryo implantation [33]. Moreover, increased IL1- $\beta$ may be due to platelet activation [34] and it decreased immediately within $24 \mathrm{~h}$. Weak release of pro-inflammatory cytokines following platelet activation has been suggested to occur after stimulation of cell proliferation, resulting in activation of tissue remodeling in vivo [35].

In vivo, hENSCs positive for SUSD2 and CD146 were found to be highly proliferative and to undergo selfrenewal in the endometrium. These cells are located around the vessels in the functionalis and basalis and participate in endometrial regeneration [4]. Moreover, SUSD2 $^{+}$hENSCs in the decidua could exhibit paracrine signaling through production of cytokines and secretomes, suggesting that they may participate in the regulation of the endometrial microenvironment [36]. In our study, the levels of SUSD2, CD146, and CD105 were increased after PRP treatment for $24 \mathrm{~h}$, suggesting the role of PRP in promoting MenSC engraftment in endometrial vascular regeneration in vivo. These results were consistent with previous studies showing that PRP-treated BMSCs could improve vascularization in bone regeneration [37, 38].

Moreover, we found that in the PRP-treated group, the expression levels of endometrial receptivity-related genes, including FoxO1, LIF, and IL1- $\beta$, were upregulated, suggesting that PRP functioned to improve endometrial function. We found that levels of FoxO1 and LIF gene expression were increased by 1.73 - and 2.68 -fold, respectively, in the $6 \mathrm{~h}$ PRP-treated group compared with that in the $6 \mathrm{~h}$ FBS-treated group. LIF is one of the most important genes affecting endometrial receptivity 
and is weakly expressed in the endometrium of patients with unexplained infertility, suggesting an important role in blastocyst implantation [39]. FoxO1 is a novel transcriptional factor involved in decidualization of endometrial stromal cells, functionally required for binding of progesterone [40]. Moreover, another previous report also demonstrated that FoxO1 is associated with vascular homeostasis in the endometrium and a mediator of the AKT pathway, suggesting an essential role in vascular and embryonic development [41]. Similar results were reported in several studies of bone and cartilage regeneration, in which PRP engraftment resulted in good outcomes and promoted the expression of particular functional genes, such as bone morphogenetic protein-2 (BMP2) and SOX9 [42]. However, HOXA10, another implantation window-related gene [43], was downregulated by PRP treatment. The mechanisms involved in this phenomenon are unclear. Thus, further studies are needed to expand on this basic in vitro study and fully elucidate the mechanisms involved in this process.

In 2012, Darzi also studied the effects of human platelets on MenSCs in vitro. He found that MenSCs are less capable of osteogenetic differentiation than BMSCs, and substitution of FBS with human platelet releasate could equalize these effects. Therefore, he suggested that human platelet releasate could be used as an osteogenic accelerator in MenSCs culture, making it a better substitute for BMSCs for bone tissue engineering [22]. In the present study, we investigated whether PRP regulated endometrial receptivity by affecting MenSCs. Our center is currently carrying out a clinical study on MenSCs transplantation as treatment of thin endometrium or intrauterine adhesions. According to our data, MenSCs transplantation can effectively increase thickness of endometrium [10], however, the pregnancy outcome is not ideal. We are committed to finding a safe and effective additive to improve the efficiency of MenSCs transplantation in curing uterine infertility. An animal study is currently ongoing to detect whether PRP affects MenSCs to improve the efficacy of transplantation in the treatment of uterine damage in vivo. We expect that autologous PRP combined with stem cell-based therapy may have excellent therapeutic effects on organic infertility and other diseases.

\section{Conclusions}

Based on their advantages of improved proliferation and non-invasiveness, MenSCs are regarded as a promising stem cell resource for regenerative medicine applications. This study showed that activated PRP could act as an excellent stimulator for MenSCs. PRP promoted MenSCs proliferation and differentiation, and elevated the expressions of SUSD2, CD146, and CD105. In addition, PRP upregulated the endometrial functionrelated targets FoxO1, LIF, and IL1- $\beta$, and downregulated the inflammation-related target IL-6 probably via phosphorylation-dependent activation of Akt and STAT3 pathways. This study is the first to indicate that PRP may regulate endometrial receptivity through effects on MenSCs. Thus, PRP may be a beneficial component in the culture of MenSCs used for endometrial regeneration therapy.

\section{Additional file}

Additional file 1: Data 1 MTS assay for cell proliferation detection. MTS assay detected proliferation of P4 MenSCs cultured with different concentrations of activated PRP or $10 \%$ FBS $(n=3)$. The data was analyzed by one-way ANOVA test, ${ }^{*} P<0.05$, ${ }^{*} P<0.01$. (TIFF $804 \mathrm{~kb}$ )

\section{Abbreviations}

ADSC: Adipose tissue-derived stromal cell; BM-MSC: Bone marrow-derived mesenchymal stromal cell; BMP2: Bone morphogenetic protein-2; CCK8: Cell counting kit-8; CFU: Colony-forming unit; CK18: Cytokeratin 18; EGF: Epidermal growth factor; FBS: Fetal bovine serum; HDP: Human platelet derivative; hENSC: Human endometrial stem cell; HGF: Hepatocyte growth factor; IGF1: Insulin-like growth factor; MenSC: Menstrual blood-derived stromal cell; MSC: Mesenchymal stem cell; OD: Optical density; PBS: phosphate-buffered saline; PDGF: Platelet-derived growth factor; PDGFR: Platelet-derived growth factor receptor; PRP: Platelet-rich plasma; RIPA: Radio immunoprecipitation assay; SUSD2: Sushi domain-containing 2; TGF $\beta-1$ : Transforming growth factor $\beta-1$; VEGF: Vascular endothelial growth factor

\section{Acknowledgements}

We are particularly grateful to Liu Cao and Yang Li for insightful comments and writing guidance. We appreciate Qiushi Wang for guidance in the preparation of the PRP. We wish to express our gratitude to Xiaoni Li for collection of menstrual blood samples and Xin Pan, Jianing Huo and Chao Han for figure guidance.

\section{Funding}

This work was supported by grants from the National Natural Science Foundation of China (81650011) and Liaoning Provincial Science and Technology Program (2015020461).

Availability of data and materials

Not applicable.

\section{Authors' contributions}

SZ was responsible for experiment conception and design, collection and assembly of data, data analysis and interpretation, and manuscript writing. $\mathrm{PL}$ revised the manuscript and provided technical guidance. $Z Y$ provided technical support. JT designed the work, provided technical guidance and final approved of manuscript. All authors read and approved the final manuscript.

Ethics approval and consent to participate

This study was approved by the Ethics Committee of the Shengjing Hospital affiliated to China Medical University (2017PS330K) and has been performed in accordance with the principles of the Declaration of Helsinki.

\section{Consent for publication}

Not applicable.

\section{Competing interests}

None declared.

\section{Publisher's Note}

Springer Nature remains neutral with regard to jurisdictional claims in published maps and institutional affiliations. 


\section{Author details}

Reproductive medicine Center, Obstetrics and Gynecology Department, Shengjing Hospital affiliated to China Medical University, No. 39 Huaxiang Road, Tiexi District, Shenyang 110022, China. ${ }^{2}$ Key Laboratory of Health Ministry for Congenital Malformation, Shengjing Hospital affiliated to China Medical University, No. 7, Economic Development Zone, Benxi 117004, China.

\section{Received: 28 July 2017 Revised: 4 December 2017}

\section{Accepted: 1 February 2018 Published online: 26 February 2018}

\section{References}

1. Meng X, Ichim TE, Zhong J, et al. Endometrial regenerative cells: a novel stem cell population. J Transl Med. 2007:5(1):1-10.

2. Gargett $\mathrm{CE}$, Masuda H. Adult stem cells in the endometrium. Mol Hum Reprod. 2010;16(11):818-34.

3. Masuda H, Anwar SS, Bühring HJ, et al. A novel marker of human endometrial mesenchymal stem-like cells. Cell Transplant. 2012;21(10):2201-14.

4. Gargett CE, Schwab KE, Deane JA. Endometrial stem/progenitor cells: the first 10 years. Hum Reprod Update. 2016;22(2):137-63.

5. Chen JY, Mou XZ, Du XC, et al. Comparative analysis of biological characteristics of adult mesenchymal stem cells with different tissue origins. Asian Pac J Trop Med. 2015;8(9):725-31.

6. Wu X, Luo Y, Chen J, et al. Transplantation of human menstrual blood progenitor cells improves hyperglycemia by promoting endogenous progenitor differentiation in type 1 diabetic mice. Stem Cells Dev. 2014;23(11):1245-57.

7. Khoury M, Alcayaga-Miranda F, Illanes SE, et al. The promising potential of menstrual stem cells for antenatal diagnosis and cell therapy. Front Immunol. 2014;5:205.

8. Borlongan CV, Kaneko Y, Maki M, et al. Menstrual blood cells display stem cell-like phenotypic markers and exert neuroprotection following transplantation in experimental stroke. Stem Cells Dev. 2010;19(4):439-52.

9. Lai $D$, Wang $F$, Yao $X$, et al. Human endometrial mesenchymal stem cells restore ovarian function through improving the renewal of germline stem cells in a mouse model of premature ovarian failure. J Transl Med. 2015;13(1):1-13.

10. Tan J, Li P, Wang Q, et al. Autologous menstrual blood-derived stromal cells transplantation for severe Asherman's syndrome. Hum Reprod. 2016;31(12): 2723-9.

11. Bu S, Wang Q, Zhang $Q$, et al. Human endometrial mesenchymal stem cells exhibit intrinsic anti-tumor properties on human epithelial ovarian cancer cells. Sci Rep. 2016;6:37019.

12. Patel AN, Park E, Kuzman M, et al. Multipotent menstrual blood stromal stem cells: isolation, characterization, and differentiation. Cell Transplant. 2008;17(3):303-11.

13. Bieback K, Hecker A, Kocaömer A, et al. Human alternatives to fetal bovine serum for the expansion of mesenchymal stromal cells from bone marrow. Stem Cells. 2009:27:2331-41.

14. Amable PR, Carias RB, Teixeira MV, et al. Platelet-rich plasma preparation for regenerative medicine: optimization and quantification of cytokines and growth factors. Stem Cell Res Ther. 2013;4(3):67.

15. Marx RE. Platelet-rich plasma (PRP): what is PRP and what is not PRP? Implant Dent. 2001;10(4):225-8.

16. Arnalich F, Rodriguez AE, Luque-Rio A, et al. Solid platelet rich plasma in corneal surgery. J Ophthalmol Ther. 2016;5(1):1-15.

17. Tobita M, Tajima S, Mizuno H. Adipose tissue-derived mesenchymal stem cells and platelet-rich plasma: stem cell transplantation methods that enhance stemness. Stem Cell Res Ther. 2015;6(1):1-7.

18. Atashi F, Jaconi ME, Pittet-Cuénod B, et al. Autologous platelet-rich plasma: a biological supplement to enhance adipose-derived mesenchymal stem cell expansion. Tissue Engineering Part C Methods. 2015;21(3):253-262.

19. Xu FT, Li HM, Yin QS, et al. Effect of activated autologous platelet-rich plasma on proliferation and osteogenic differentiation of human adiposederived stem cells in vitro. Am J Transl Res. 2014;7(2):257-70.

20. Masoudi E, Ribas J, Kaushik G, et al. Platelet-rich blood derivatives for stem cellbased tissue engineering and regeneration. Curr Stem Cell Rep. 2016;2(1):33-42.

21. do Amaral RJ, Da SN, Haddad NF, et al. Platelet-rich plasma obtained with different anticoagulants and their effect on platelet numbers and mesenchymal stromal cells behavior in vitro. Stem Cells Int. 2016;2016(3):7414036.

22. Darzi S, Zarnani A H, Jedditehrani M, et al. Osteogenic differentiation of stem cells derived from menstrual blood versus bone marrow in the presence of human platelet releasate. Tissue Eng Part A. 2012;18(15-16): 1720-1728.
23. Kazemnejad S, Najafi R, Zarnani AH, et al. Comparative effect of human platelet derivatives on proliferation and osteogenic differentiation of menstrual blood-derived stem cells. Mol Biotechnol. 2014;56(3):223-31.

24. Chen J, Du X, Chen Q, et al. Effects of donors' age and passage number on the biological characteristics of menstrual blood-derived stem cells. Int $J$ Clin Exp Pathol. 2015;8(11):14584-95.

25. Verdi J, Tan A, Shoae-Hassani A, et al. Endometrial stem cells in regenerative medicine. J Biol Eng. 2014;8(1):20.

26 Marmotti A, Rossi R, Castoldi F, et al. PRP and articular cartilage: a clinical update. Biomed Res Int. 2015;542502

27 Zhou Y. The differential effects of leukocyte-containing and pure platelet-rich plasma (PRP) on tendon stem/progenitor cells - implications of PRP application for the clinical treatment of tendon injuries. Stem Cell Res Ther. 2015:6(1):173.

28 Fernandes G, Yang S. Application of platelet-rich plasma with stem cells in bone and periodontal tissue engineering. Bone Research. 2016:4:16036.

29 Li Q, Pan S, Dangaria SJ, et al. Platelet-rich fibrin promotes periodontal regeneration and enhances alveolar bone augmentation. J BioMed Res Int 2013;2013(10):638043.

30 Amable PR, Teixeira MV, Carias RB, et al. Mesenchymal stromal cell proliferation, gene expression and protein production in human platelet-rich plasmasupplemented media. PLoS One. 2014;9(8):e104662.

31 Yin W, Xin Q, Zhang Y, et al. Advantages of pure platelet-rich plasma compared with leukocyte- and platelet-rich plasma in promoting repair of bone defects. J Transl Med. 2016:14(1):73.

32 Griffith OW, Chavan AR, Protopapas S, et al. Embryo implantation evolved from an ancestral inflammatory attachment reaction. Proc Natl Acad Sci U S A. 2017;114(32):E6566

33 Laird SM, Tuckerman EM, Li TC. Cytokine expression in the endometrium of women with implantation failure and recurrent miscarriage. Reprod Biomed Online. 2006;13(1):13-23.

34 Bendinelli P, Matteucci E, Dogliotti G, et al. Molecular basis of antiinflammatory action of platelet-rich plasma on human chondrocytes: mechanisms of NF-kB inhibition via HGF. J Cell Physiol. 2010;225(3):757-66.

35 Luo $S$, Wang $Y, A n Q$, et al. Platelets protect lung from injury induced by systemic inflammatory response platelets protect lung from injury induced by systemic inflammatory response. J Sci Rep. 2017;7:42080. https://doi.org/ 10.1038/srep42080

36 Murakami K, Lee YH, Lucas ES, et al. Decidualization induces a secretome switch in perivascular niche cells of the human endometrium. J Endocrinol. 2014;155(11):4542-53.

37 Kasten $\mathrm{P}$, Beverungen $\mathrm{M}$, Lorenz $\mathrm{H}$, et al. Comparison of platelet-rich plasma and VEGF-transfected mesenchymal stem cells on vascularization and bone formation in a critical-size bone defect. Cells Tissues Organs. 2012;196(6):523-33.

38 Zhang ZY, Huang AW, Fan JJ, et al. The potential use of allogeneic plateletrich plasma for large bone defect treatment: immunogenicity and defect healing efficacy. J Cell Transplantation. 2013;22(1):175-87.

39 Cui N, Wang C, Zhao Z, et al. The roles of anandamide, fatty acid amide hydrolase, and leukemia inhibitory factor on the endometrium during the implantation window. Front Endocrinol. 2017;8:268.

40 Vasquez YM, Mazur EC, Li X, et al. FOXO1 is required for binding of PR on IRF4, novel transcriptional regulator of endometrial stromal decidualization. Mol Endocrinol. 2015;29(3):421-33.

41 Dharaneeswaran H, Abid MR, Yuan L, et al. FOXO1-mediated activation of Akt plays a critical role in vascular homeostasis. Circ Res. 2014;115(2):238-51.

42 Salamanna F, Veronesi F, Maglio M, et al. New and emerging strategies in platelet-rich plasma application in musculoskeletal regenerative procedures: general overview on still open questions and outlook. Biomed Res Int. 2015;2015:846045

43 Jana SK, Banerjee P, Mukherjee R, et al. HOXA-11 mediated dysregulation of matrix remodeling during implantation window in women with endometriosis. J Assist Reprod Genet. 2013:30(11):1505. 